\title{
Laparoscopic treatment of pancreatic trauma in children: A case reports
}

\author{
de-Vos C*, Sidler D and Moore S \\ Division of Paediatric Surgery, Department of Surgical Sciences, Faculty of Health Sciences, University of Stellenbosch, Tygerberg Children’s Hospital, South Africa
}

\begin{abstract}
The most common cause of pancreatic injury in children is blunt abdominal trauma. The appropriate treatment is controversial. There are two schools of thought: conservative (Gr I-V) treatment or surgical treatment (Gr III-V). Laparoscopic treatment for Gr III and IV injury is becoming increasingly more popular. We report on 2 patients which sustained pancreatic transection from blunt abdominal trauma, where both were successfully treated with a laparoscopic distal pancreatectomy. Our preliminary experience shows that laparoscopic treatment of pancreatic transection is safe and feasible.
\end{abstract}

\section{Introduction}

Pancreatic injury is rare in children [1]. The most common cause is blunt abdominal trauma from a bicycle handle bar. Classical presentation includes epigastric pain accompanied by episodes of vomiting [2]. The appropriate treatment is controversial. There are two schools of thought; conservative (Gr I-IV) or surgical treatment (Gr III-V). Laparoscopic treatment, particularly for grade III and IV injury, is increasingly becoming popular for children.

Risk of complications depends on severity of injury and integrity of the pancreatic duct $[3,4]$. Early laparoscopic treatment of Gr III - IV injury could likely avert such complications.

\section{Methods}

We present two patients who sustained a pancreatic injury after blunt abdominal trauma, one through the body and tail and the second one through the tai only. The injuries were graded according to the American Association of Surgeons' Trauma scoring scale (Table 1).

The first patient is a 12-year-old boy, who presented with a history of falling over the handle bar of his bicycle a day before admission. $\mathrm{He}$ had one episode of vomiting and complained of generalized abdominal pain. His amylase and lipase were both marginally raised (Table 1). Patient 2, a 3-year-old boy, presented on the day of injury after being involved in a pediastrian vehicle accident. He had 2 episodes of vomiting, abdominal distention and generalized abdominal tenderness. Most of his blood results were within normal limits (Table 2) except for his amylase, ALT and AST that were marginally raised.

A CT abdomen in patient 1 showed a grade III pancreatic injury (full thickness laceration through body and tail of the pancreas) with extensive peripancreatic, intra-abdominal and pelvic free fluid (Figure 1). Splenic vessels and all other intra-abdominal organs were intact.

The second patient had a full thickness laceration involving the tail of the pancreas (Figure 2). In addition, he had multiple liver, involving segments $6 \& 7$ as well as the caudate lobe and splenic lacerations (Figure 3). The splenic vessels seemed intact.
Table 1. Pancreas Injury Scale [4]

\begin{tabular}{|l|l|l|}
\hline Grade & Type of injury & Description of Injury \\
\hline I & Hematoma & Minor contusion without duct injury \\
\hline & Laceration & Superficial laceration without duct injury \\
\hline II & Hematoma & Major contusion without duct injury of tissue loss \\
\hline & Laceration & Major laceration without duct injury or tissue loss \\
\hline III & Laceration & Distal transection or parenchymal injury with duct injury \\
\hline IV & Laceration & Proximal transection or parenchymal injury involving ampulla \\
\hline V & Laceration & Massive disruption of pancreatic head \\
\hline
\end{tabular}

Table 2. Labatory result

\begin{tabular}{|c|c|c|c|c|c|}
\hline PATIENT & S-AMYLASE & LIPASE & HB & U\&E & LFT \\
\hline Patient 1 & 540 & 264 & 12.2 & normal & - \\
\hline Patient 2 & 253 & - & 13.2 & normal & $\begin{array}{c}\text { AST }=360 \\
\text { ALT }=175\end{array}$ \\
\hline
\end{tabular}

Both patients had an emergency laparoscopic distal pancreatectomy on the day of admission. Indication for surgery was based on the findings of an acute abdomen, the massive amount of peripancreatic, intra-abdominal and pelvic free fluid leaving an uncertainty that bowel could be damaged. The pancreatic duct wasn't visualized in either patient. In the first patient the pancreatic stump was oversew and a drain left in situ. In the second patient Glubran 2 was sprayed to seal the pancreatic duct.

\section{Result}

Both patients had an excellent post-operative recovery with a short hospital stay ( $5 \& 7$ days). Patient 2 received 5 days of TPN, tolerated a full ward diet on day 6 and was discharge on day 7. A followup ultrasound at 6 weeks showed no abnormality in both patients,

*Correspondence to: de-Vos C, Division of Paediatric Surgery, Department of Surgical Sciences, Faculty of Health Sciences, University of Stellenbosch, Tygerberg Children's Hospital, South Africa, E-mail: devos.corne@gmail.com

Received: February 01, 2019; Accepted: February 08, 2019; Published: February 11,2019 

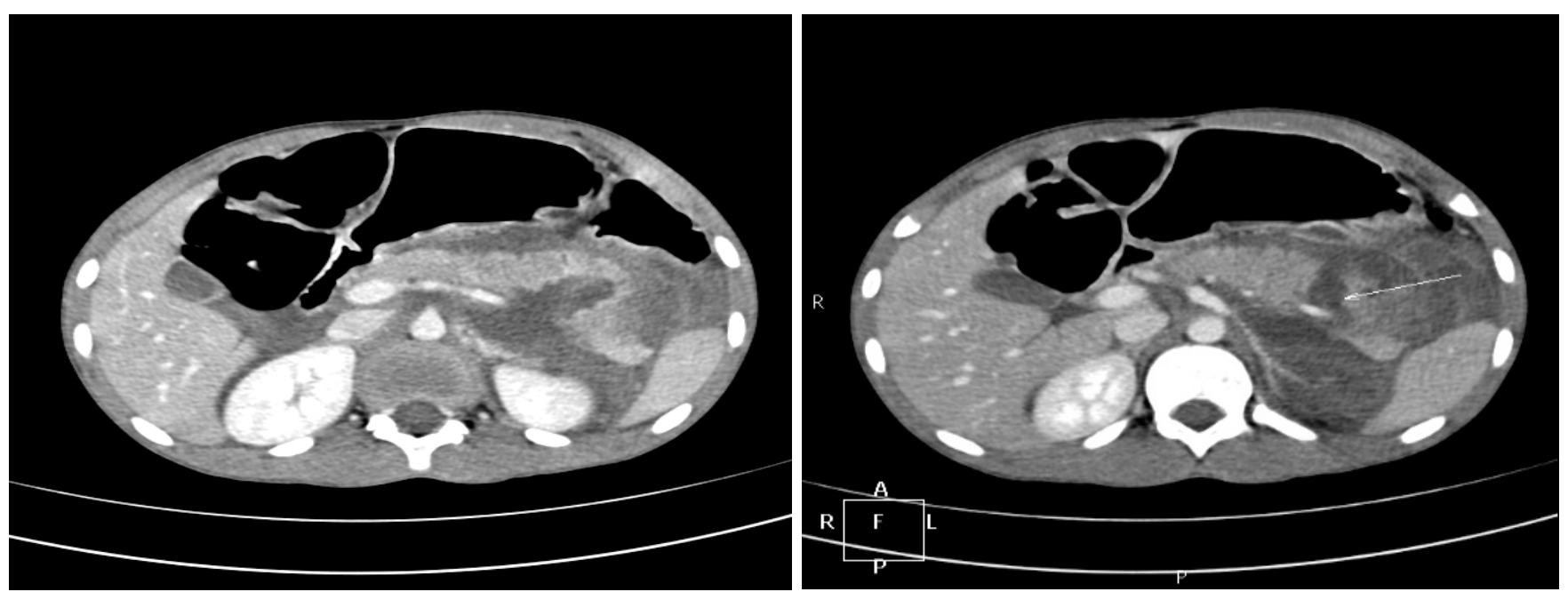

Figure 1. Extensive peripancreatic, intra-abdominal and pelvic free fluid

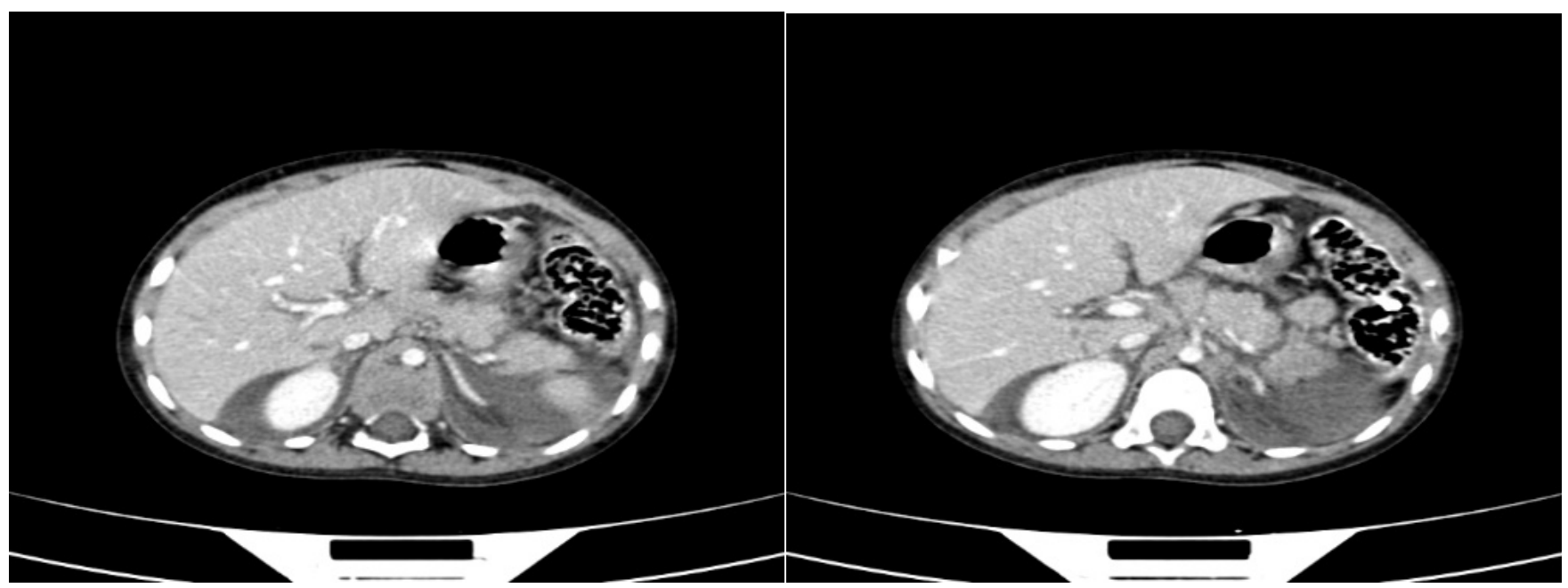

Figure 2. Laceration involving the tail of the pancreas
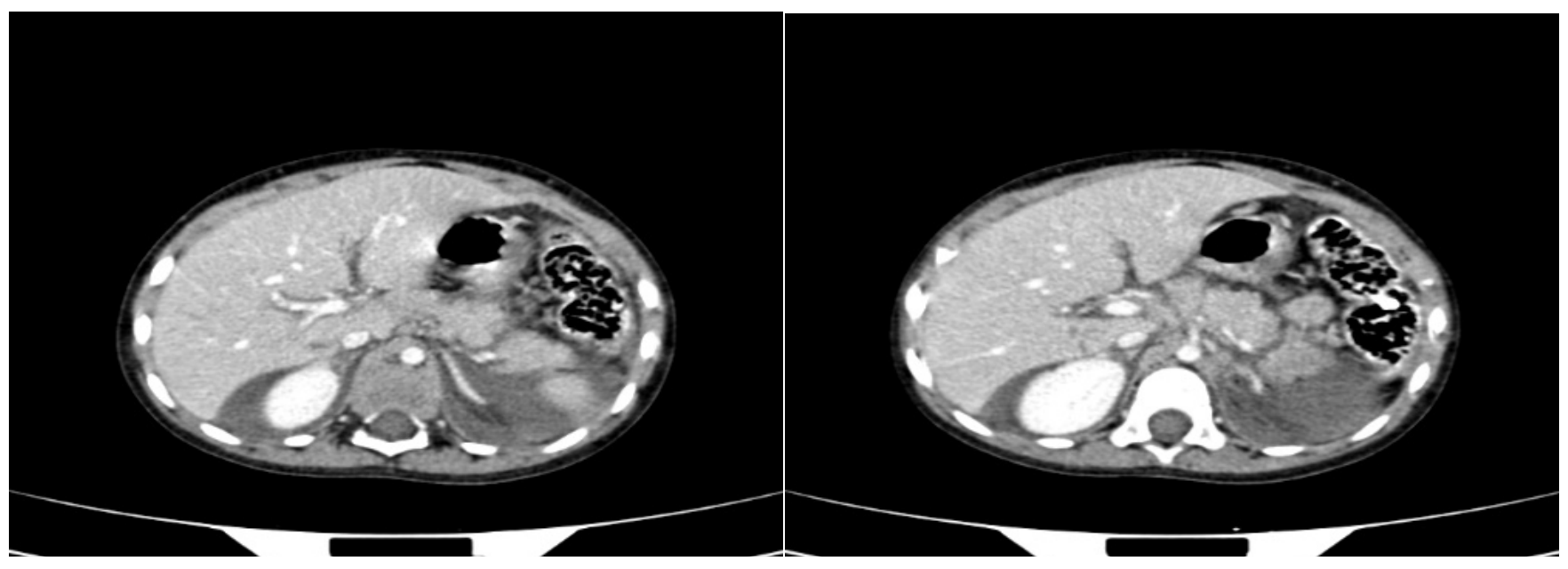

Figure 3. Splenic and liver lacerations 


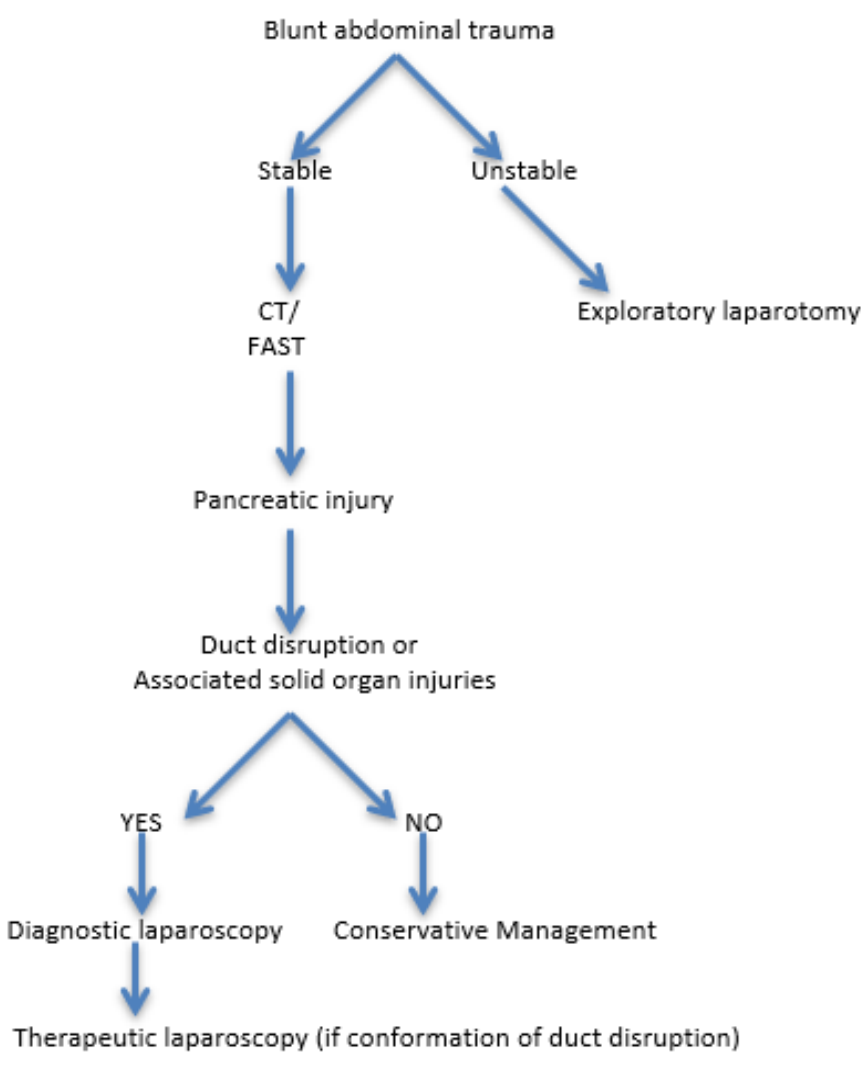

Figure 4. Proposed adapted algoritm for treatment of pancreatic injuries

particularly no pseudo-cyst. On a 3 months follow up both patients were asymptomatic with a normal glucose profile.

\section{Discussion}

Although laparoscopic treatment for pancreatic injury is relatively uncommon and new in the paediatric patient population, it has been well described in adults [5]. There are two opposing views, some favour a conservative approach, others who are laparoscopic supporters a surgical approach. ${ }^{3}$ An advantage of the laparoscopic technique is the potential to perserve the spleen [6]. Those surgeons who advocate a conservative approach in the acute setting would suggest an ERCP some time later to assess the integrity of the pancreatic duct [7]. If transsection of the pancreas is present, the duct can either be stented or after a distal pancreatectomy the stump oversewn or stapled. We used successfully glue (Glubran 2) in one patient. Many of these patients, particulary with Gr III-IV injury, have a high morbidity from pseudocysts needing repeated interventions that could lead to a prolonged hospital stay. Our preliminary experience supports that early interverntion, such as a laparoscopic distal pancreatectomy, is a good alterntive to conservative management of pancreatic duct disrubtions and seems to reduce morbidty and hospital stay. We find Numanoglu's flow chart (Figure 4) [2] very useful.

\section{Conclusion}

Although the surgical treatment of pancreatic blunt trauma with duct disruption is controversial, our prelimanary experience with 2 patients shows that laparoscopic distal pancreatectomy is feasable, a well tolerated and an alternative to the conservative approach with a low morbidity that should be increasingly used [2] especially when spleen-sparing surgery in early grade III injuries is intended [1]. The post-operative recovery seems is much quicker with a shorter hospital stay. Futher experience with more paediatric patients is necessary before a definitive conclusion can be drawn.

\section{References}

1. Stringer MD (2005) Pancreatic trauma in children. Br J Surg 92: 467-470. [Crossref]

2. Numonoglu A (2010) Laparoscopy in abdominal trauma. CME 28: 119-121.

3. Pujahari AK (2012) Laparoscopic non-resectional management of Gr III isolated pancreatic injury. Tropical Gastroenterology 33: 148-150.

4. Takaori K, Tanigawa N (2007) Laparoscopic pancreatic resection: the past, present, and future. Surg Today 37: 535-545. [Crossref]

5. Rutkoski JD, Segura BJ, Kane TD (2011) Experience with totally laparoscopic distal pancreatectomy with splenic preservation for pediatric trauma-2 techniques. $J$ Pediatr Surg 46: 588-93. [Crosssref]

6. Leva E, Huscher C, Rode H, Fava G, Napolitano M, et al (2008) Management of traumatic complete pancreatic fracture in a child: case report and review of literature. $J$ Laparoendosc Adv Surg Tech A 18: 321-323. [Crossref]

7. Al-Jazaeri AH (2011) Short hospitalization after early intervention in mangement of Gr III pancreatic injuries in children: A possible trend. Saudi J gastroenterology 17: 5.

Copyright: ( 2019 de-Vos C. This is an open-access article distributed under the terms of the Creative Commons Attribution License, which permits unrestricted use, distribution, and reproduction in any medium, provided the original author and source are credited. 